\title{
Esophageal POEM: the new standard of care
}

\author{
Salmaan Jawaid, Peter V. Draganov, Dennis Yang \\ Division of Gastroenterology, Hepatology, and Nutrition, University of Florida Health, Gainesville, FL, USA \\ Contributions: (I) Conception and design: S Jawaid, D Yang; (II) Administrative support: None; (III) Provision of study materials or patients: None; \\ (IV) Collection and assembly of data: None; (V) Data analysis and interpretation: None; (VI) Manuscript writing: All authors; (VII) Final approval of \\ manuscript: All authors. \\ Correspondence to: Dennis Yang, MD. Division of Gastroenterology, Hepatology, and Nutrition, University of Florida Health, 1329 SW 16 th St, \\ Room 5262, Gainesville, FL 32608, USA. Email: dennis.yang@medicine.ufl.edu.
}

\begin{abstract}
Since its introduction into clinical practice nearly a decade ago, per-oral endoscopic myotomy (POEM) has now become an accepted minimally invasive approach for the treatment of achalasia with excellent short- and mid-term clinical outcomes in both treatment naïve and those who have failed prior therapy. POEM is comparable to laparoscopic Heller myotomy (LHM) in terms of efficacy and safety, with less procedural pain and faster recovery time. Recent data also reveals that POEM may be more effective and durable than pneumatic dilation (PD) for the treatment of achalasia, with similar safety profile. Preliminary data on POEM for spastic esophageal disorders (SED) is promising yet scarce. Post-POEM gastroesophageal reflux disease (GERD) is common but asymptomatic in most patients, further highlighting the need for ongoing research in this field and the importance of long-term surveillance of these patients.
\end{abstract}

Keywords: Per-oral endoscopic myotomy (POEM); achalasia; laparoscopic Heller myotomy (LHM); esophageal motility disorder; pneumatic dilation (PD); dysphagia; Eckardt

Received: 10 October 2019; Accepted: 19 December 2019; Published: 05 October 2020.

doi: $10.21037 / \operatorname{tgh} .2019 .12 .17$

View this article at: http://dx.doi.org/10.21037/tgh.2019.12.17

\section{Introduction}

Achalasia is an idiopathic esophageal motility disorder resulting from degeneration of the myenteric plexus of the esophageal wall, ultimately leading to esophageal aperistalsis and impaired relaxation of the lower esophageal sphincter (LES), the two hallmark features seen on diagnostic testing with high-resolution manometry (HRM) $(1,2)$. Diagnosis is based on HRM, and a high index of suspicion in patients with typical symptoms (dysphagia, regurgitation, chest pain, weight loss), endoscopic and radiographic findings. Treatment revolves around improving esophageal emptying by mechanically disrupting the LES.

Traditionally, laparoscopic Heller myotomy (LHM) and pneumatic dilation (PD) were the two main modalities for the treatment of symptomatic achalasia patients (3). With the advent of third space endoscopy, per-oral endoscopic myotomy (POEM) was introduced a decade ago as an alternate minimally invasive approach for these patients $(4,5)$ and has thereafter become an established supported therapy given its excellent and consistent clinical outcomes.

\section{Technique}

\section{Patient preparation}

Food retention in the esophagus is common among patients with achalasia. While there is no consensus, maintaining patients on clear liquids 24 hours prior to the procedure and fasting overnight is often sufficient for adequate clearance (6). However, in patients with dilated sigmoid esophagus, several days of clear liquids and prolonged fasting may be required for proper preparation. In all, we recommend performing POEM cases using general anesthesia and endotracheal intubation to help minimize the risk of aspiration during the procedure (6). The use of air has been associated with significant adverse events, including but not limited to pain related to pneumomediastinum, gas 

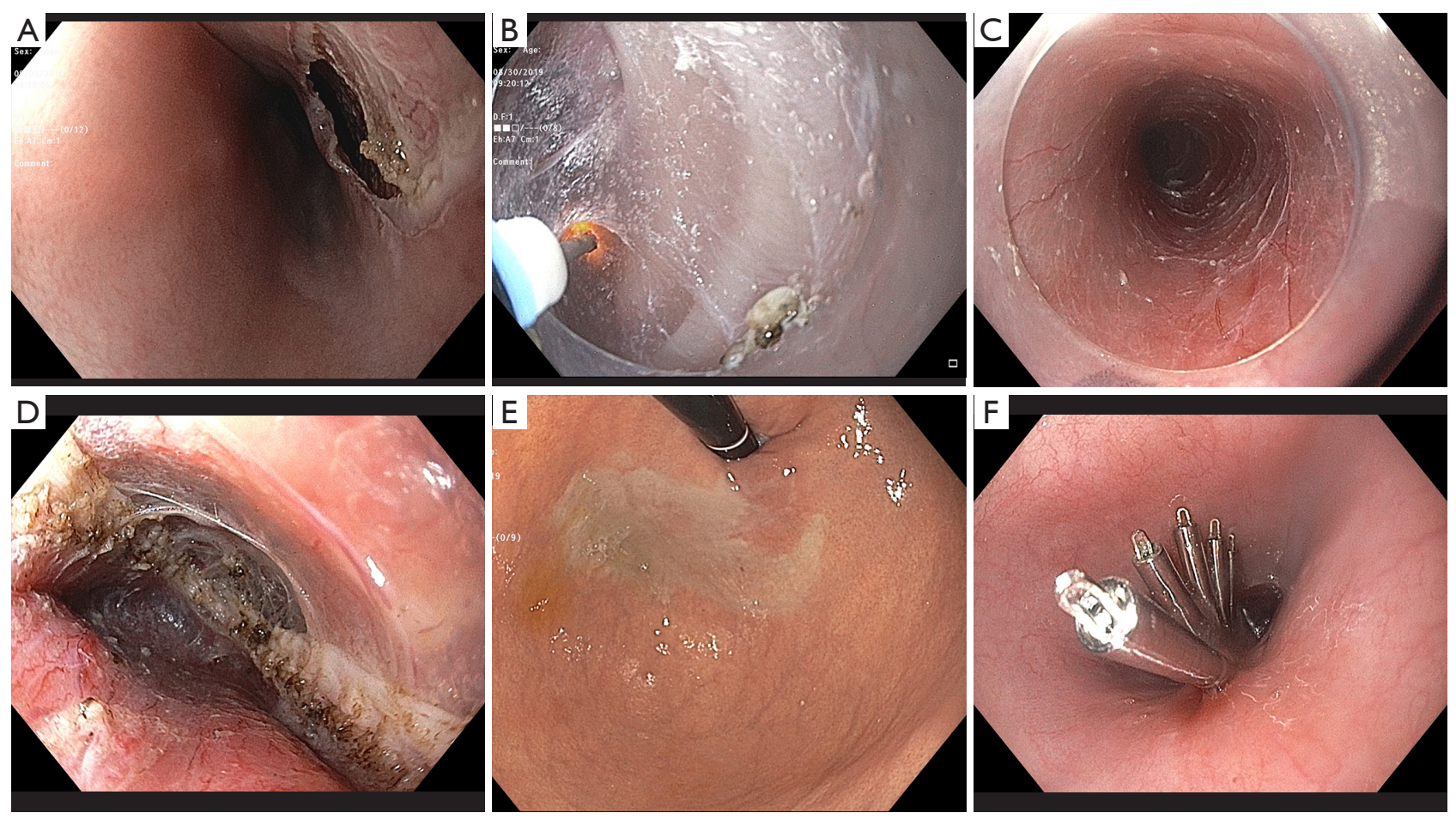

Figure 1 Steps involved in POEM. (A) Initial mucosal incision; (B) submucosal tunneling during POEM; (C) completed submucosal dissection; (D) endoscopic view of completed myotomy; (E) evidence of adequate myotomy demonstrated by a loose LES and blanching caused by epinephrine injected at the end of the tunnel; (F) clip closure of mucosal incision. POEM, per-oral endoscopic myotomy; LES, lower esophageal sphincter.

embolism, abdominal compartment syndrome, and cardiac arrest. Thus, POEM should be strictly performed with carbon dioxide $\left(\mathrm{CO}_{2}\right)$ insufflation (7-9).

\section{Mucosotomy}

After attachment of a transparent distal cap, the endoscope is inserted and the gastroesophageal junction (GEJ) is identified. Mucosal incision is then typically performed 14-15 cm proximal to the GEJ with the instillation of $8-10 \mathrm{~mL}$ of submucosal injectate to create a cushion, followed by a $15 \mathrm{~mm}$ longitudinal mucosal incision (Figure 1A) using the electrocautery knife. When creating the submucosal bleb, care must be taken to avoid superficial injection and lifting of the space between the mucosa and muscularis mucosa instead of the intended deeper submucosa. Under-recognition of this step can lead to esophageal intramural dissection $(10,11)$. Once a submucosal lift has been attained and the initial mucosal incision performed, the edges of the mucosotomy should be carefully "trimmed" with the electrocautery knife. This step is important as to ensure adequate widening of the mucosotomy site for safe scope insertion and reduces the risk of bleeding from mechanical trauma of superficial vessels at the incision edges.

\section{Submucosal tunneling}

Mucosal incision and subsequent submucosal tunneling during POEM can be performed by adopting either an anterior or posterior approach. With an anterior approach, the initial incision is often made in the 2 o'clock position in the right anterolateral esophagus, as initially described by Inoue (5). This permits the creation of a straight submucosal tunnel terminating at the anterior aspect of the lesser curvature. With the posterior approach, the mucosal incision is typically made at the 5 to 6 o'clock orientation with the device/accessories in the same axis as the direction of the submucosal tunnel. Previous meta-analysis of randomized controlled trials (RCTs) demonstrated no difference in overall clinical efficacy or incidence of gastroesophageal reflux disease (GERD) between the 
anterior $v s$. posterior approach (12-14). More recently, Khashab et al. randomized patients to receive an anterior or posterior myotomy and found similar rates of clinical success and abnormal acid exposure (49\% in anterior group $v s .42 \%$ in the posterior group, $\mathrm{P}=0.67$ ) (13). The posterior approach can be advantageous in patients with previous surgical myotomy by avoiding scar tissue typically seen in the post-surgical anterior plane $(15,16)$.

Submucosal dissection can be carried out by using a combination of dissection with the electrocautery knife (Figure $1 B, C$ ) and repeated injection of dyed saline solution. It is important to maintain spatial orientation during submucosal tunneling and recognize the esophageal wall layers as to avoid inadvertent injury to the underlying mucosa. The submucosal tunnel is then extended typically $2-3 \mathrm{~cm}$ into the gastric cardia below the esophagogastric junction (EGJ). Assessing adequate tunnel length can be performed through various techniques. We favor injecting $1 \mathrm{~mL}$ of 1:10,000 epinephrine into the submucosal space at the termination of the submucosal tunnel followed by visualization of blanching seen at the cardia while in the retroflexed position within the stomach. Alternatively, a second ultraslim endoscope can be inserted into the stomach and retroflexed while the standard gastroscope is within the submucosal tunnel, allowing assessment of the exact extent of the tunnel via transillumination (17). Estimation of the length of the submucosal tunnel by identifying landmarks under fluoroscopic marking has also been reported (18). Regardless of the method chosen, adequate extension of the tunnel into the cardia is crucial in order to ensure subsequent complete esophagogastric myotomy.

\section{Myotomy}

Myotomy is typically begun $2-3 \mathrm{~cm}$ distal to the initial mucosotomy site. We generally attempt to maintain the integrity of the longitudinal muscle layer by performing selective myotomy of the circular muscle fibers (partial myotomy) in the esophagus but dissection of both the circular and longitudinal muscles (complete full-thickness myotomy) at the EGJ and cardia (Figure 1D). Both studies by Li et al. and Duan et al. have demonstrated no difference in clinical efficacy or GERD related symptoms between partial and full-thickness myotomy, although the former approach had shorter procedure times $(19,20)$. One recent small retrospective study suggests concurrent myotomy and submucosal tunneling after performing a partial tunnel, may be more efficient than standard full tunnel followed by selective myotomy (21). However, most experts will perform the full submucosal tunnel prior to the myotomy. The adequate length of the myotomy is still under investigation but likely should be tailored based on disease characteristics, including findings on high-resolution manometry (HRM) (22). Intraprocedural determination of adequate myotomy is typically performed by ease of passage of the endoscope through the EGJ on the luminal side and visual confirmation of complete myotomy (Figure $1 E$ ). Few studies have shown that the use of a real-time imaging probe (EndoFLIP) can objectively measure effects of myotomy on EGJ diameter and distensibility, which may potentially provide objective parameters regarding the adequacy and completeness of the myotomy (23).

\section{Closure}

Adequate closure of the initial mucosotomy site is crucial to prevent contamination of the peritoneal/mediastinal cavity. Both endoscopic suturing and clip (Figure $1 F$ ) are effective means of endoscopic closure (24). The over the scope clip (OTSC) is an alternative closure method if mucosotomy closure is technically challenging $(25,26)$.

\section{Clinical outcomes of POEM for the treatment of achalasia}

\section{Short term outcomes}

Since its inception over a decade ago, more than 7,000 POEM cases have been performed worldwide (27). Numerous studies have demonstrated excellent and consistent short-term clinical outcomes. In a recent metaanalysis of 36 studies and 2,373 patients, Akintoye et al. reported that POEM achieved clinical success, defined by an Eckardt score $\leq 3$, in $98 \%$ of the patients (95\% CI, 97-100\%) with sustained response at 12-month followup (28). Other comprehensive systemic reviews and metaanalysis have further confirmed similar clinical efficacy based on subjective and objective parameters, with significant decreases in Eckardt scores, lower esophageal sphincter (LES) pressures, and barium retention rates on esophagrams (29-32).

\section{Mid-term outcomes}

Data on the efficacy of POEM has continued to accumulate over recent years. The largest systemic review performed 
by Li et al. evaluated outcomes of POEM in patients with achalasia (median follow-up interval of 30 months). A total of 10 studies and 373 patients were ultimately included with clinical success achieved in $92.9 \%$. Mean Eckardt scores decreased from 7.4 to 1.4 post myotomy and mean LES pressures decreased from 32.8 to $13.7 \mathrm{mmHg}$ post myotomy (33). Similarly, in a prospective study of 500 patients, Inoue et al. also reported sustained clinical response based on Eckardt scores and LES pressures $(34,35)$. In the longest follow-up studies to date (5 years), both Teitelbaum et al. (36) and Zhang et al. (37) demonstrated excellent sustained clinical efficacy (between 83-95\%). These studies collectively further support the initial excellent clinical outcomes of POEM and its mid-term durability. Long-term outcomes are eagerly being awaited.

\section{Safety profile}

POEM has also been associated with an excellent safety profile. In the meta-analysis performed by Akintoye et al., the incidence of serious adverse events was low, with mucosal perforation and clinically significant bleeding being reported in only $0.7 \%$ and $0.2 \%$, respectively (28). While post-procedural pain is commonly reported, most patients can be easily managed with oral pain medications and need for long-term narcotics is infrequently required (9). In fact, Benias et al. followed 62 consecutive patients who underwent POEM and demonstrated the most common indication for hospitalization was post-procedure pain. However, post-procedure pain was not a predictor of poor outcomes and many patients in their cohort were not routinely hospitalized (38). While there is still no consensus on the optimal immediate post-procedural follow-up protocol, many expert centers will routinely perform oral contrast studies to rule out complications. In our single-center prospective study, we demonstrated that CT esophagram allowed the prompt recognition of a perforation not identified during POEM in an otherwise stable patient without clinical manifestations (39). Future studies are needed to further identify a cost-effective postprocedural monitoring strategy.

\section{POEM in patients after prior failed therapy}

Recurrence of symptoms after LHM has been estimated to occur in $10-20 \%$ of patients $(40,41)$. Performing a repeat $\mathrm{HM}$ can prove to be challenging in this patient population due to significant fibrosis and scarring (42). While PD has been shown to be an effective salvage therapy in these patients (43), relapse is high and multiple sessions are often required to induce long-term remission (44). In addition, each PD puts the patient at risk for potentially serious adverse events, most notably esophageal perforation.

POEM has been recently evaluated as a potential alternative for patients with previously failed LHM. With POEM, the endoscopist can select the most appropriate orientation of the myotomy and circumvent the prior surgical site. Furthermore, during POEM, the myotomy can be further extended in the mid and proximal esophagus, which may be of benefit in patients with type III achalasia. In 2018, Tyberg et al. performed the first prospective multicenter, international study evaluating the use of POEM as a salvage technique. Technical success was achieved in $100 \%$ of patients with clinical success seen in $94 \%$ of patients at 12 -month follow-up. Adverse events occurred in $13 \%$ of patients but were managed either conservatively or through endoscopic means (45). In another multicenter study, POEM also achieved a high clinical response rate in patients with prior LHM, albeit lower in comparison to treatment naïve patients $(81 \% v s$. 94\%; $\mathrm{P}=0.01$ ) with no difference in adverse events (46). In a more recent study, Zhang et al. found a high clinical response rate for POEM in patients with previous LHM (95.7\%) at median 28-month follow-up (37). In aggregate, POEM appears to be a reasonable alternative to redo LHM in patients with persistent/recurrent symptoms, although current data are limited to observational studies.

\section{POEM compared to LHM and PD for achalasia}

Traditionally, LHM was considered the primary treatment modality for patients with achalasia. Initial studies comparing both POEM and LHM showed similar shortterm clinical efficacy and rate of adverse events, albeit limited by their single-center design $(47,48)$. In a 2015 retrospective multicenter study comparing both treatment modalities, Kumbhari et al. demonstrated a higher clinical response rate $(98.0 \%$ POEM vs. $80.8 \%$ LHM, $\mathrm{P}=0.01)$ and lower rate of adverse events (6\% POEM vs. 27\% LHM, $\mathrm{P}<0.01)$ in patients undergoing POEM. Moreover, despite a longer myotomy (16 cm POEM vs. $8 \mathrm{~cm}$ LHM, $\mathrm{P}<0.01$ ), POEM required significantly less time than LHM (102 vs. 264 min; $\mathrm{P}<0.01$ ) (49). Similar clinical efficacy was seen in a very recent meta-analysis of 1,213 patients comparing both treatment modalities, with lower postoperative Eckardt scores in the POEM group compared to LHM 
(pooled standardized mean difference $-0.58 ; 95 \%$ CI, -1.03 to -0.13). Interestingly, there was no difference in reflux symptoms and pathologic reflux on $\mathrm{pH}$ monitoring between both groups (50). A more recent study by Schlottmann et al. showed patients undergoing POEM were more likely to have improvement of dysphagia at 12 months (93.5\% for POEM and $91.0 \%$ for LHM, P=0.01) and 24 months (92.7\% for POEM and $90.0 \%$ for LHM, P=0.01), but significantly more GERD and erosive esophagitis (OR, 9.31; 95\% CI, 4.71-18.85; $\mathrm{P}<0.0001)(51)$. Given its minimally invasive approach, POEM has been associated with better patient tolerability when compared to LHM. Docimo et al. successfully demonstrated patients undergoing POEM require less narcotic use for pain control $(35.8 \mathrm{mg}$ POEM vs. $101.8 \mathrm{mg}$ LHM, $\mathrm{P}<0.001)$ with a consequent shorter length of hospital stay (31.2 hrs POEM and 55.79 hrs LHM, $\mathrm{P}<0.0001$ ) (52). Data from randomized clinical trials between POEM and LHM are lacking with only one small study recently published in abstract form, demonstrating similar clinical response and adverse event rates between the two treatment modalities (53).

Initial observational studies comparing POEM and PD for the treatment of achalasia suggested higher response rates with POEM for all achalasia subtypes (54). More recently, Ponds et al. reported results of their randomized clinical trial comparing POEM vs. PD in achalasia patients. This study demonstrated that POEM was associated with a higher sustained response when compared to PD (92\% vs. 54\%, $\mathrm{P}<0.01$ ) at 24 months (55). There were no serious adverse events reported with POEM whereas one perforation occurred after PD. Overall, more patients in the POEM group were maintained on acid suppression therapy given the higher rate of reflux esophagitis ( $41 \%$ in POEM vs. $7 \%$ in $\mathrm{PD}, \mathrm{P}=0.02)$; albeit most of these were mild in nature (LA grade $\mathrm{A}$ or $\mathrm{B}$ ).

The introduction and widespread adoption of HRM and the development of the Chicago Classification system have allowed the revision of esophageal motility disorders (1). It is now well accepted that achalasia encompasses different subtypes, with each characterized by a distinct esophageal contractility pattern. With this development, several studies have further evaluated the efficacy of achalasia treatment options in the context of this disease spectrum. While previous studies suggested significant variation in clinical response of LHM and PD based on subtype of achalasia (56), accumulating data suggest that POEM is highly effective for all three achalasia phenotypes, particularly for achalasia type III (57). A recent comparative study identified 64 and
177 patients who underwent POEM and PD, respectively. At the 24 months follow-up period, the clinical success rate of POEM was much higher than PD across all subtypes, although only type I and type II were statistically significant (type 1: $92.0 \%$ vs. $51.1 \%, \mathrm{P}=0.004$; type $2: 92.3 \%$ vs. $59.8 \%$, $\mathrm{P}=0.007$; and type $3: 91.7 \%$ vs. $55.6 \%, \mathrm{P}=0.051$ ) (58). A recent meta-analysis comparing outcomes of treatment for achalasia for all manometric subtypes, evaluated 1,575 patients and found that POEM was more likely to be successful than LHM for both type I (OR, 2.97; 95\% CI, 1.09 to $8.03 ; \mathrm{P}=0.032)$ and type III $(\mathrm{OR}, 3.50 ; 95 \% \mathrm{CI}, 1.39$ to $8.77 ; \mathrm{P}=0.007)$ achalasia. The likelihood of success of POEM and LHM for type II achalasia was similar (59). This data further highlights the importance of targeted therapy and underscores a potentially unique feature of POEM: the ability to tailor the length of the myotomy based on disease characteristics.

\section{POEM for the treatment of spastic esophageal disorders (SED) and esophagogastric junction outflow obstruction (EGJOO)}

SED include spastic achalasia (type III), diffuse esophageal spasm, and jackhammer (JH, hypercontractile) esophagus. Most of these patients will classically present with both chest pain and dysphagia. The pathophysiology for SED is typically rooted in a hypertensive esophageal body wall in addition to a noncompliant LES (minimal relaxation of the LES) (60). Up to this point, providing definitive therapy for this patient population has been limited and management focused primarily on symptom control. In 2013, Vanuytsel et al. performed a RCT demonstrating the short term ( $<6$ months) effectiveness of Botulinum toxin for patients with distal esophageal spasm (DES) and hypercontractile esophagus. However, this primarily included patients with dysphagia, and not chest pain, as the predominant symptom (61). Conceptually, performing myotomy of both the mid esophageal body (where hypertensive contractions occur) as well as LES, may help alleviate both symptoms. To do so would require a longer myotomy into the thoracic esophagus, which is typically technically difficult in LHM but technically feasible with POEM. Khashab et al. retrospectively showed POEMs effectiveness in reducing both dysphagia (93\%) and chest pain symptoms (87\%) in patients with SED who failed medical therapy (62). The study was limited by its relatively short-term follow-up (8 months). Notably, when stratified based on subtypes of SED, symptoms improved more significantly in the 
achalasia type III (96.3\%) and DES (100\%) then when compared to $\mathrm{JH}$ esophagus (70\%) $(\mathrm{P}=0.05)$. A subsequent systemic review and meta-analysis corroborated these findings and found a $20 \%$ difference in weighted pooled response rates after POEM was performed in patients with achalasia III vs. JH esophagus (92\% in achalasia III and $72 \%$ in $\mathrm{JH}, \mathrm{P}=0.01$ ) (63). The etiology for the lower response rates in patients with $\mathrm{JH}$ esophagus remains unclear, as a recent study by Chandan $e t a l$. demonstrated that the length of the myotomy (more $v s$. less than $10 \mathrm{~cm}$ ) during POEM did not impact clinical outcomes (64). In aggregate, the current data on POEM for SED is promising; yet scarce, heterogenous and lacking long-term follow-up. Future prospective trials are needed to further evaluate the role of POEM in the management of this difficult to treat population.

With the widespread adoption of HRM, a possible variant of achalasia, commonly referred as EGJOO, has been recently identified. EGJOO is characterized by elevated integrated relaxation pressure (IRP) but normal peristalsis on HRM with no alternate cause (65). Data regarding management of these patients has been scarce and primarily in the form of observational and case studies. Previous case series on Botulinum toxin injection and PD have shown variable response rates ranging between $61 \%$ to $79 \%(66,67)$. More recently, Khashab et al. reported outcomes of POEM for the treatment of non-achalasia esophageal motility disorders in 50 patients (68), of which 15 carried a diagnosis of EGJOO. POEM for EGJOO was associated with a clinical success of $93 \%$ at a median followup of 195 days with normalization of IRP on post-POEM HRM in $71 \%$ of the patients. While limited by the small numbers, this data suggests a possible role of POEM for these patients and the need for larger prospective studies to corroborate these initial findings.

\section{POEM and GERD}

Gastroesophageal reflux is a common sequela following therapy aimed at disrupting the LES barrier. Risk of abnormal acid reflux (based on 24-hour $\mathrm{pH}$ studies) after PD has been estimated to occur in $15-27 \%$ of patients $(41,69)$. Furthermore, GERD has also been commonly reported after LHM, even in those following fundoplication. Indeed, when objective parameters such as $\mathrm{pH}$ studies are used, reflux rates in patients post-LHM have been estimated as high as $42 \%$ (70).

Initial studies evaluating acid reflux after POEM demonstrated low incidence of GERD (between 6-15\%) (71). However, most of these studies used symptoms as a surrogate marker for reflux and were primarily originating from Asia. Subsequent studies employing objective parameters (endoscopic evidence of reflux esophagitis or 24-hour $\mathrm{pH}$ studies) have demonstrated a higher incidence of abnormal acid exposure following POEM. In a study of 282 patients postPOEM, of which $83 \%$ had follow-up endoscopy, reflux esophagitis (mostly LA grade A or B) was identified in $23.2 \%$ of patients of which $60.1 \%$ were asymptomatic (72). Akintoye's meta-analysis demonstrated the rates of symptomatic gastroesophageal reflux, esophagitis on esophagogastroduodenoscopy (EGD), and abnormal acid exposure after POEM (based on $\mathrm{pH}$ monitoring) were $8.5 \%$ (95\% CI, $4.9-13 \%$ ), $13 \%$ (95\% CI, $5.0-23 \%$ ), and $47 \%$ (95\% CI, 21-74\%), respectively. A recent meta-analysis evaluating the risk of GERD in 1,581 POEM patients, demonstrated that abnormal acid exposure was identified on $39.0 \%$ (95\% CI, 24.5-55.8\%) on $\mathrm{pH}$ monitoring and reflux esophagitis present in $29.4 \%$ (95\% CI, $18.5-43.3 \%$ ) (73).

Overall, it is clear that GERD remains a common issue following therapies for esophageal motility disorders aimed at disrupting the hypertensive LES. Importantly, the literature demonstrates that the majority of patients are asymptomatic and thereby highlight the importance of routine surveillance. Most of these patients are adequately managed with acid suppressive therapy (e.g., proton pump inhibitors); yet long-term follow-up is necessary to elucidate the long-lasting effects of such strategy. In light of this issue, there has been an increasing interest on potential endoscopic anti-reflux measures following POEM. A small prospective study by Tyberg et al. demonstrated that transoral incisionless fundoplication is technically feasible and potentially helpful for the prevention of GERD postPOEM (74). More recently, Inoue et al. introduced the concept of endoscopic fundoplication following POEM by successfully creating a mechanical barrier from within the peritoneal cavity in 21 patients (75). Future studies are needed to determine potential risk factors for GERD after POEM and to identify those patients in whom additional anti-reflux therapies may be necessary.

\section{Conclusions}

POEM is no longer a novel experimental therapy, but rather an established first-line treatment for symptomatic achalasia. POEM is superior to LHM for certain achalasia subtypes and is a more durable treatment when compared to 
PD. While POEM appears to be effective for the treatment of other SED; data remains limited and long-term followup is needed. GERD is the most common adverse effect following POEM and surveillance is important given that most patients remain asymptomatic. Future research is necessary to determine the best long-term strategy for the management of post-POEM patients with GERD.

\section{Acknowledgments}

None

\section{Footnote}

Conflicts of Interest: The authors have no conflicts of interest to declare.

Ethical Statement: The authors are accountable for all aspects of the work in ensuring that questions related to the accuracy or integrity of any part of the work are appropriately investigated and resolved.

\section{References}

1. Vaezi MF, Pandolfino JE, Vela MF. ACG clinical guideline: diagnosis and management of achalasia. Am J Gastroenterol 2013;108:1238-49; quiz 1250.

2. Goldblum JR, Whyte RI, Orringer MB, et al. Achalasia. A morphologic study of 42 resected specimens. Am J Surg Pathol 1994;18:327-37.

3. Schoenberg MB, Marx S, Kersten JF, et al. Laparoscopic Heller myotomy versus endoscopic balloon dilatation for the treatment of achalasia: a network meta-analysis. Ann Surg 2013;258:943-52.

4. Pasricha PJ, Hawari R, Ahmed I, et al. Submucosal endoscopic esophageal myotomy: a novel experimental approach for the treatment of achalasia. Endoscopy 2007;39:761-4.

5. Inoue H, Minami H, Kobayashi $Y$, et al. Peroral endoscopic myotomy (POEM) for esophageal achalasia. Endoscopy 2010;42:265-71.

6. Yang D, Pannu D, Zhang Q, et al. Evaluation of anesthesia management, feasibility and efficacy of peroral endoscopic myotomy (POEM) for achalasia performed in the endoscopy unit. Endosc Int Open 2015;3:E289-95.

7. Maher SZ, Chintanaboina J, Eun Kim D, et al. Pneumopericardium complicating per-oral endoscopic myotomy due to inadvertent use of air instead of carbon dioxide. ACG Case Rep J 2018;5:e59.

8. Zhang XC, Zhou PH. Sa1000 major peri-operative complications of peroral endoscopic myotomy (POEM): experience based on 1680 patients. Gastrointes Endo 2016;83:AB198.

9. Misra L, Fukami N, Nikolic K, et al. Peroral endoscopic myotomy: procedural complications and pain management for the perioperative clinician. Med Devices (Auckl) 2017;10:53-9.

10. Ye L, Jiang J, Ngamruengphong S, et al. Intramural esophageal dissection during peroral endoscopic myotomy. Endoscopy 2019;51:E197-8.

11. Yang D, Draganov PV. Tips and tricks for avoiding the dreaded intramural esophageal dissection during peroral endoscopic myotomy: recognize! Endoscopy 2019;51:996.

12. Rodríguez de Santiago E, Mohammed N, Manolakis A, et al. Anterior versus posterior myotomy during poem for the treatment of achalasia: systematic review and meta-analysis of randomized clinical trials. J Gastrointestin Liver Dis 2019;28:107-15.

13. Khashab MA, Sanaei O, Rivory J, et al. Peroral endoscopic myotomy: anterior versus posterior approach: a randomized single-blinded clinical trial. Gastrointest Endosc 2020;91:288-97.e7.

14. Tan Y, Lv L, Wang X, et al. Efficacy of anterior versus posterior per-oral endoscopic myotomy for treating achalasia: a randomized, prospective study. Gastrointest Endosc 2018;88:46-54.

15. Stavropoulos SN, Modayil RJ, Friedel D, et al. The International Per Oral Endoscopic Myotomy Survey (IPOEMS): a snapshot of the global POEM experience. Surg Endosc 2013;27:3322-38.

16. Onimaru M, Inoue $\mathrm{H}$, Ikeda $\mathrm{H}$, et al. Peroral endoscopic myotomy is a viable option for failed surgical esophagocardiomyotomy instead of redo surgical Heller myotomy: a single center prospective study. J Am Coll Surg 2013;217:598-605.

17. Grimes KL, Inoue H, Onimaru M, et al. Doublescope per oral endoscopic myotomy (POEM): a prospectiverandomized controlled trial. Surg Endosc 2016;30:1344-51.

18. Kumbhari V, Besharati S, Abdelgelil A, et al. Intraprocedural fluoroscopy to determine the extent of the cardiomyotomy during per-oral endoscopic myotomy (with video). Gastrointest Endosc 2015;81:1451-6.

19. Li C, Gong A, Zhang J, et al. Clinical outcomes and safety of partial full-thickness myotomy versus circular muscle myotomy in peroral endoscopic myotomy for achalasia 
patients. Gastroenterol Res Pract 2017;2017:2676513.

20. Duan T, Tan Y, Zhou J, et al. A retrospective study of peroral endoscopic full-thickness myotomy in patients with severe achalasia. J Laparoendosc Adv Surg Tech A 2017;27:770-6.

21. Philips GM, Dacha S, Keilin SA, et al. Concurrent myotomy and tunneling after establishment of a half tunnel instead of myotomy after establishment of a full tunnel: a more efficient method of peroral endoscopic myotomy. Endosc Int Open 2016;4:E403-8.

22. Chang KJ. Endoscopic foregut surgery and interventions: the future is now. The state-of-the-art and my personal journey. World J Gastroenterol 2019;25:1-41.

23. Campagna RAJ, Carlson DA, Hungness ES, et al. Intraoperative assessment of esophageal motility using FLIP during myotomy for achalasia. Surg Endosc 2019. [Epub ahead of print].

24. Grimes KL, Inoue H. Per oral endoscopic myotomy for achalasia: a detailed description of the technique and review of the literature. Thorac Surg Clin 2016;26:147-62.

25. Yang D, Draganov PV. Closing the gap in POEM. Endoscopy 2013;45:677.

26. Saxena P, Chavez YH, Kord Valeshabad A, et al. An alternative method for mucosal flap closure during peroral endoscopic myotomy using an over-the-scope clipping device. Endoscopy 2013;45:579-81.

27. Swanström LL. POEM outcomes: how long is long enough? Gastrointest Endosc 2017;85:934-5.

28. Akintoye E, Kumar N, Obaitan I, et al. Peroral endoscopic myotomy: a meta-analysis. Endoscopy 2016;48:1059-68.

29. Barbieri LA, Hassan C, Rosati R, et al. Systematic review and meta-analysis: efficacy and safety of POEM for achalasia. United European Gastroenterol J 2015;3:325-34.

30. Crespin OM, Liu LWC, Parmar A, et al. Safety and efficacy of POEM for treatment of achalasia: a systematic review of the literature. Surg Endosc 2017;31:2187-201.

31. Patel K, Abbassi-Ghadi N, Markar S, et al. Peroral endoscopic myotomy for the treatment of esophageal achalasia: systematic review and pooled analysis. Dis Esophagus 2016;29:807-19.

32. Evensen H, Kristensen V, Larssen L, et al. Outcome of peroral endoscopic myotomy (POEM) in treatmentnaive patients. A systematic review. Scand J Gastroenterol 2019;54:1-7.

33. Li H, Peng W, Huang S, et al. The 2 years' long-term efficacy and safety of peroral endoscopic myotomy for the treatment of achalasia: a systematic review. J Cardiothorac Surg 2019;14:1.
34. Inoue H, Sato H, Ikeda H, et al. Per-oral endoscopic myotomy: a series of 500 patients. J Am Coll Surg 2015;221:256-64.

35. Ngamruengphong S, Inoue H, Chiu PW, et al. Long-term outcomes of per-oral endoscopic myotomy in patients with achalasia with a minimum follow-up of 2 years: an international multicenter study. Gastrointest Endosc 2017;85:927-33.e2.

36. Teitelbaum EN, Dunst CM, Reavis KM, et al. Clinical outcomes five years after POEM for treatment of primary esophageal motility disorders. Surg Endosc 2018;32:421-7.

37. Zhang X, Modayil RJ, Friedel D, et al. Per-oral endoscopic myotomy in patients with or without prior Heller's myotomy: comparing long-term outcomes in a large U.S. single-center cohort (with videos). Gastrointest Endosc 2018;87:972-85.

38. Benias PC, Korrapati P, Inamdar S, et al. Tu1186 safety and feasibility of performing outpatient poem with same day discharge and factors that predict success. Gastrointest Endosc 2018;87:AB560.

39. Pannu D, Yang D, Abbitt PL, et al. Prospective evaluation of CT esophagram findings after peroral endoscopic myotomy. Gastrointest Endosc 2016;84:408-15.

40. Moonen A, Annese V, Belmans A, et al. Long-term results of the European achalasia trial: a multicentre randomised controlled trial comparing pneumatic dilation versus laparoscopic Heller myotomy. Gut 2016;65:732-9.

41. Boeckxstaens GE, Annese V, des Varannes SB, et al. Pneumatic dilation versus laparoscopic Heller's myotomy for idiopathic achalasia. N Engl J Med 2011;364:1807-16.

42. Wang L, Li YM. Recurrent achalasia treated with Heller myotomy: a review of the literature. World J Gastroenterol 2008;14:7122-6.

43. Kumbhari V, Behary J, Szczesniak M, et al. Efficacy and safety of pneumatic dilation for achalasia in the treatment of post-myotomy symptom relapse. Am J Gastroenterol 2013;108:1076-81.

44. Legros L, Ropert A, Brochard C, et al. Long-term results of pneumatic dilatation for relapsing symptoms of achalasia after Heller myotomy. Neurogastroenterol Motil 2014;26:1248-55.

45. Tyberg A, Sharaiha RZ, Famillary P. Peroral endoscopic myotomy as salvation technique post-Heller: international experience. Dig Endosc 2018;30:52-6.

46. Ngamruengphong S, Inoue H, Ujiki MB, et al. Efficacy and safety of peroral endoscopic myotomy for treatment of achalasia after failed Heller myotomy. Clin Gastroenterol Hepatol 2017;15:1531-7.e3. 
47. Bhayani NH, Kurian AA, Dunst CM, et al. A comparative study on comprehensive, objective outcomes of laparoscopic Heller myotomy with per-oral endoscopic myotomy (POEM) for achalasia. Ann Surg 2014;259:1098-103.

48. Chan SM, Wu JC, Teoh AY, et al. Comparison of early outcomes and quality of life after laparoscopic Heller's cardiomyotomy to peroral endoscopic myotomy for treatment of achalasia. Dig Endosc 2016;28:27-32.

49. Kumbhari V, Tieu AH, Onimaru M, et al. Peroral endoscopic myotomy (POEM) vs laparoscopic Heller myotomy (LHM) for the treatment of type III achalasia in 75 patients: a multicenter comparative study. Endosc Int Open 2015;3:E195-201.

50. Park CH, Jung DH, Kim DH, et al. Comparative efficacy of per-oral endoscopic myotomy and Heller myotomy in patients with achalasia: a meta-analysis. Gastrointest Endosc 2019;90:546-58.e3.

51. Schlottmann F, Luckett DJ, Fine J, et al. Laparoscopic Heller myotomy versus peroral endoscopic myotomy (POEM) for achalasia: a systematic review and metaanalysis. Ann Surg 2018;267:451-60.

52. Docimo S Jr, Mathew A, Shope AJ, et al. Reduced postoperative pain scores and narcotic use favor per-oral endoscopic myotomy over laparoscopic Heller myotomy. Surg Endosc 2017;31:795-800.

53. Moura ET, Farias GF, Coutinho LM, et al. 472 A randomized controlled trial comparing peroral endoscopic myotomy (POEM) versus laparoscopic Heller myotomy with fundoplication in the treatment of achalasia. Gastrointest Endosc 2019;89:AB84.

54. Meng F, Li P, Wang Y, et al. Peroral endoscopic myotomy compared with pneumatic dilation for newly diagnosed achalasia. Surg Endosc 2017;31:4665-72.

55. Ponds FA, Fockens P, Lei A, et al. Effect of peroral endoscopic myotomy vs pneumatic dilation on symptom severity and treatment outcomes among treatment-naïve patients with achalasia: a randomized clinical trial. JAMA 2019;322:134-44.

56. Rohof WO, Salvador R, Annese V, et al. Outcomes of treatment for achalasia depend on manometric subtype. Gastroenterology 2013;144:718-25; quiz e13-4.

57. Kim WH, Cho JY, Ko WJ, et al. Comparison of the outcomes of peroral endoscopic myotomy for achalasia according to manometric subtype. Gut Liver 2017;11:642-7.

58. Kim GH, Jung KW, Jung HY, et al. Superior clinical outcomes of peroral endoscopic myotomy compared with balloon dilation in all achalasia subtypes. J Gastroenterol Hepatol 2019;34:659-65.

59. Andolfi C, Fisichella PM. Meta-analysis of clinical outcome after treatment for achalasia based on manometric subtypes. Br J Surg 2019;106:332-41.

60. Roman S, Kahrilas PJ. Management of spastic esophageal disorders. Gastroenterol Clin North Am 2013;42:27-43.

61. Vanuytsel T, Bisschops R, Farré R, et al. Botulinum toxin reduces dysphagia in patients with nonachalasia primary esophageal motility disorders. Clin Gastroenterol Hepatol 2013;11:1115-21.e2.

62. Khashab MA, Messallam AA, Onimaru M, et al. International multicenter experience with peroral endoscopic myotomy for the treatment of spastic esophageal disorders refractory to medical therapy (with video). Gastrointest Endosc 2015;81:1170-7.

63. Khan MA, Kumbhari V, Ngamruengphong S, et al. Is POEM the answer for management of spastic esophageal disorders? A systematic review and meta-analysis. Dig Dis Sci 2017;62:35-44.

64. Chandan S, Mohan BP, Chandan OC, et al. Clinical efficacy of per-oral endoscopic myotomy (POEM) for spastic esophageal disorders: a systematic review and metaanalysis. Surg Endosc 2020;34:707-18.

65. Scherer JR, Kwiatek MA, Soper NJ, et al. Functional esophagogastric junction obstruction with intact peristalsis: a heterogeneous syndrome sometimes akin to achalasia. J Gastrointest Surg 2009;13:2219-25.

66. Richter JE, Clayton SB. Diagnosis and management of esophagogastric junction outflow obstruction. Am J Gastroenterol 2019;114:544-7.

67. Clayton SB, Shin CM, Ewing A, et al. Pneumatic dilation improves esophageal emptying and symptoms in patients with idiopathic esophago-gastric junction outflow obstruction. Neurogastroenterol Motil 2019;31:e13522.

68. Khashab MA, Familiari P, Draganov PV, et al. Peroral endoscopic myotomy is effective and safe in non-achalasia esophageal motility disorders: an international multicenter study. Endosc Int Open 2018;6:E1031-6.

69. Min YW, Lee JH, Min BH, et al. Association between gastroesophageal reflux disease after pneumatic balloon dilatation and clinical course in patients with achalasia. J Neurogastroenterol Motil 2014;20:212-8.

70. Rawlings A, Soper NJ, Oelschlager B, et al. Laparoscopic Dor versus Toupet fundoplication following Heller myotomy for achalasia: results of a multicenter, prospective, randomized-controlled trial. Surg Endosc 2012;26:18-26. 
71. Callahan ZM, Su B, Ujiki M. Management of reflux after peroral endoscopic myotomy. J Xiangya Med 2019;4:6.

72. Kumbhari V, Familiari P, Bjerregaard NC, et al. Gastroesophageal reflux after peroral endoscopic myotomy: a multicenter case-control study. Endoscopy 2017;49:634-42.

73. Repici A, Fuccio L, Maselli R, et al. GERD after per-oral endoscopic myotomy as compared with Heller's myotomy with fundoplication: a systematic review with meta-

doi: $10.21037 / \operatorname{tgh} .2019 .12 .17$

Cite this article as: Jawaid S, Draganov PV, Yang D. Esophageal POEM: the new standard of care. Transl Gastroenterol Hepatol 2020;5:47. analysis. Gastrointest Endosc 2018;87:934-43.e18.

74. Tyberg A, Choi A, Gaidhane M, et al. Transoral incisional fundoplication for reflux after peroral endoscopic myotomy: a crucial addition to our arsenal. Endosc Int Open 2018;6:E549-52.

75. Inoue H, Ueno A, Shimamura $\mathrm{Y}$, et al. Peroral endoscopic myotomy and fundoplication: a novel NOTES procedure. Endoscopy 2019;51:161-4. 